\title{
Coexistence of Two Singularities in Dewetting Flows: Regularizing the Corner Tip
}

\author{
Ivo Peters ${ }^{1,2}$, Jacco H. Snoeijer ${ }^{2}$, Adrian Daerr ${ }^{1}$, Laurent Limat ${ }^{1}$ \\ ${ }^{1}$ Laboratoire Matière et Systèmes Complexes, UMR CNRS 705\%, Université Paris Diderot, \\ 10 rue Alice Domon et Léonie Duquet, 75205 Paris cedex 13, France \\ ${ }^{2}$ Physics of Fluids Group and J. M. Burgers Centre for Fluid Dynamics, \\ University of Twente, P.O. Box 217, 7500 AE Enschede, The Netherlands
}

(Dated: July 17, 2009)

\begin{abstract}
Entrainment in wetting and dewetting flows often occurs through the formation of a corner with a very sharp tip. This corner singularity comes on top of the divergence of viscous stress near the contact line, which is only regularized at molecular scales. We investigate the fine structure of corners appearing at the rear of sliding drops. Experiments reveal a sudden decrease of tip radius, down to 20 microns, before entrainment occurs. We propose a lubrication model for this phenomenon, which compares well to experiments. Despite the disparity of length scales, it turns out that the tip size is set by the classical viscous singularity, for which we deduce a nanometric length from our macroscopic measurements.
\end{abstract}

Fluid interfaces can be deformed into singular structures exhibiting length scales much smaller than that of the global flow. The most common example is a water drop detaching from a faucet, developing a singularity at pinch-off $[1,2]$. Similar topological changes occur when the flow near the interface is driven so strongly that one of the fluid phases can invade the other. This so-called entrainment often occurs through a sharp cusp or tip [3-7], as is e.g. observed for air bubbles entrained by a jet or solid plunging into a liquid pool [8-12]. However, below the critical driving strength the interface remains at steady state and a stationary, singular structure is formed. Besides fundamental interest, this control over small length scales is crucial in applications such as spray formation and inkjet printing $[13,14]$, while entrainment is rate limiting in coating [15].

A peculiar situation arises in wetting flows, when the liquid is bounded by a corner-shaped contact line [810, 16-18], cf Fig. 1b. Above a critical speed the sharp corner tip breaks up to entrain bubbles or droplets depending on whether the contact line is advancing or receding. This corner singularity emerges on top of the famous moving contact line singularity: even a perfectly straight contact line develops diverging viscous stress when maintaining a no-slip boundary condition down to molecular scale [19, 20]. Despite progress on the flow away from the tip [21, 22], it has remained unclear how these two singularities can coexist, whether they are related and what determines the sharpness of the corner tip [17].

In this Letter we investigate the fine structure of corner tips appearing at the rear of drops sliding down an inclined plane (Fig. 1). The steady-state corners are characterized by the tip radius $R$, which close to the entrainment threshold is found to decrease dramatically with drop speed $U$. Using a lubrication model we derive the approximate relation

$$
R=\ell e^{\theta_{e}^{3} / 9 \mathrm{Ca}},
$$

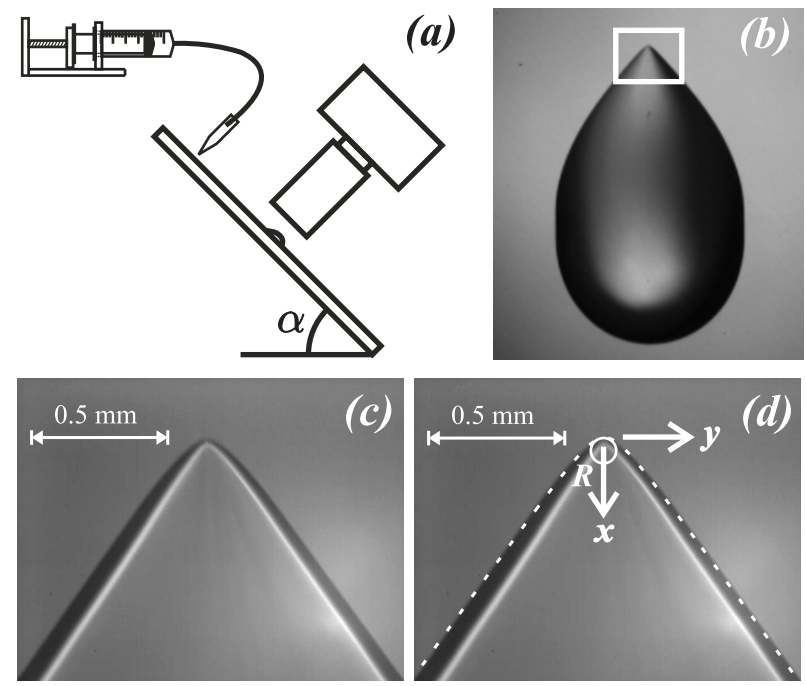

FIG. 1: (a) Sketch of the experimental set-up. Partially wetting silicone oil drops slide down an inclined plane with constant velocity. (b) The interface shape of the drop is monitored from above for different sliding velocities. At large speeds a sharp corner forms at the rear of the drop. (c) The tip radius $R$ can be determined from a zoom of the corner tip. (d) Same as in (c) showing that the contact line is well approximated by a hyperbolic shape (dashed, using $R=50 \mu \mathrm{m}$ ).

that accurately describes the experimental observations. Here $\theta_{e}$ is the equilibrium (receding) contact angle and the speed dependence appears through the capillary number $\mathrm{Ca}=U \eta / \gamma$, where $\eta$ and $\gamma$ denote viscosity and surface tension. We identify the length $\ell$ as the molecular scale associated with the microscopic physics of wetting [23-31]. We obtain a length of the order of $10 \mathrm{~nm}$ by fitting the experimental data. From a hydrodynamic point of view, this is the scale at which the classical viscous singularity is regularized.

Dewetting corners thus have the remarkable feature that the tip size is governed by an inner length scale $\ell$, much smaller than the tip radius itself. This scenario 


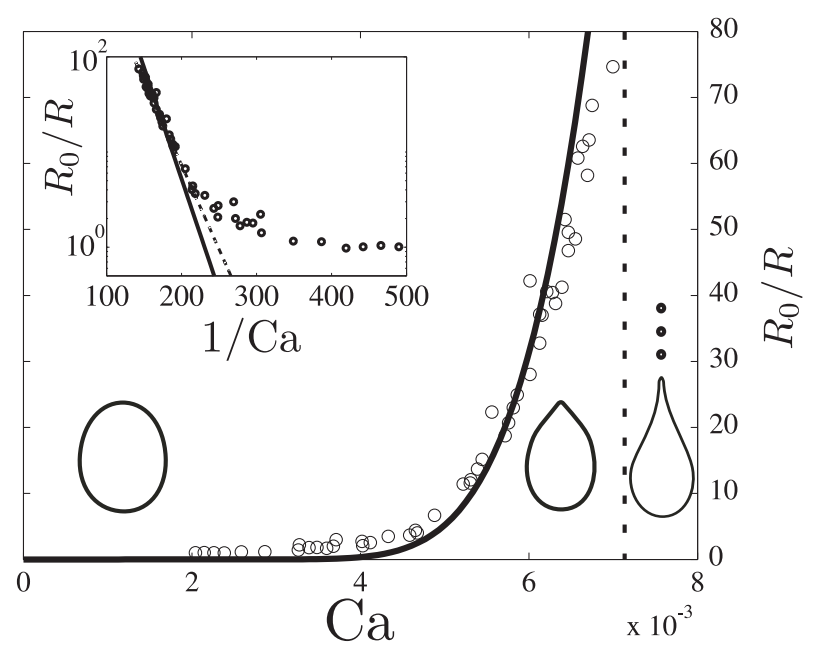

FIG. 2: Experimental measurements of the tip curvature $1 / R$ as a function of Ca. Data are normalized by the contact line radius at zero speed, $R_{0}=1.63 \mathrm{~mm}$. At low Ca the curvature stays nearly constant $R / R_{0} \approx 1$, while close to the pearling transition (vertical dashed line) the curvature increases nearly two orders of magnitude. Solid line indicates the prediction (1) with $\ell=7 \mathrm{~nm}$. Inset: The logarithmic plot confirms the predicted scaling. Dashed line is the best linear fit.

is very different from free surface singularities without a contact line, even though these can exhibit a similar exponentially decreasing tip size. For example, the cusp solution by Jeong \& Moffat [3] scales as $R_{\text {cusp }} \sim L e^{-\mathrm{Ca}}$, but in this case $L$ is an outer length scale characterizing the macroscopic flow.

Experiments. - A schematic view of the experimental setup is given in Fig. 1a. Silicon oil drops are deposited on an inclined glass plate $(\eta=18.6 \mathrm{cP}, \gamma=$ $\left.0.0205 \mathrm{~N} / \mathrm{m}, \rho=940 \mathrm{~kg} / \mathrm{m}^{3}\right)$. The drops detach from a pipette connected to a syringe pump, resulting in a constant drop volume (typically $8-10 \mathrm{~mm}^{3}$ ). The drops slide down at a constant speed $U$ that is controlled by the angle at which the plate is inclined. The glass plate is coated with fluoro-polymers (FC725), providing partial wetting conditions for silicon oil [16] with static advancing and receding angles of $55^{\circ}$ and $45^{\circ}$ respectively. As we consider the receding contact line at the rear of the drop we take $\theta_{e}=45^{\circ}$. The corners are visualized with large magnification that is achieved by using a $25 \mathrm{~mm}$ Pentax lens in reversed direction combined with several macro extension tubes. The optical resolution of the images like Fig. 1c on which the actual tip curvature measurements are done is $2 \mu \mathrm{m} /$ pixel on a 1 Mega pixel image.

Figure 2 shows experimental results on the contact line curvature $1 / R$ as a function of drop speed Ca. Tip radii are normalized by the radius of curvature of a static drop of the same volume, $R_{0}=1.63 \mathrm{~mm}$. At low speeds the curvature remains constant, while a rapid increase of the curvature can be seen at capillary numbers $\mathrm{Ca} \gtrsim 5 \cdot 10^{-3}$. This behavior coincides with the onset of the cornered

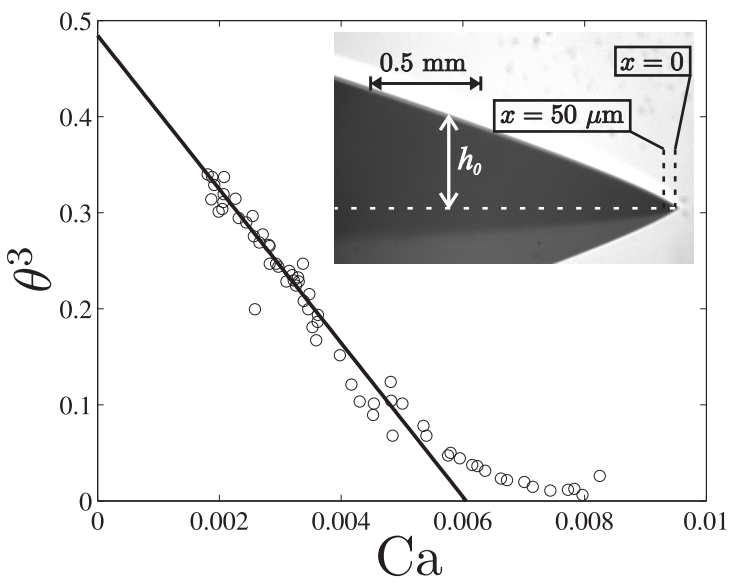

FIG. 3: The receding contact angle $\theta$ measured at a fixed distance $x=50 \mu \mathrm{m}$. A linear fit is made to the Cox-Voinov regime (solid line). A clear departure from the linear regime sets in at $\mathrm{Ca} \approx 6 \cdot 10^{-3}$, where $R$ approaches the measurement scale of $50 \mu \mathrm{m}$. Inset: A side view of the rear of a drop sliding from right to left. $h_{0}$ is the height of the drop along the centerline.

shape. The measurements continue up to the "pearling transition' at which small droplets are entrained, occurring around $\mathrm{Ca}=7 \cdot 10^{-3}$. The smallest tip size we find before this entrainment is approximately $20 \mu \mathrm{m}$, which is nearly two orders of magnitude smaller than the global drop size. The scaling (1) is revealed in the inset of Fig. 2, showing the curvature $1 / R$ on a semi-logarithmic scale versus $1 / \mathrm{Ca}$. In the corner regime the data agree very well with this exponential behavior. The solid line was fitted using the length scale $\ell$ as the sole adjustable parameter, yielding $\ell=7 \mathrm{~nm}$. We wish to emphasize, however, that the determination of $\ell$ is very sensitive to the details of the fit. For example, when fitting (1) using $\theta_{e}$ as second adjustable parameter one finds $\theta_{e}=41^{\circ}$ and $\ell=65 \mathrm{~nm}$ (dashed line). We nevertheless conclude that the length scale is of nanometric size, consistent with the typical size of silicone oil molecules [17].

We now wish to demonstrate that $\ell$ is related to the regularization of the viscous singularity that appears in the Cox-Voinov law for the dynamic contact angle $\theta[23$, 24 ,

$$
\theta^{3}=\theta_{e}^{3}-9 \mathrm{Ca} \ln x / \ell_{\theta}
$$

This dynamic angle varies logarithmically with the distance to the contact line $x$, cut-off at a scale $\ell_{\theta}$. The precise interpretation of this length depends on the physics at molecular scale, which goes beyond hydrodynamics and beyond the purpose of the present paper [23-31]. Here we estimate $\ell_{\theta}$ by measuring the contact angle along the central axis of the drop, very near the tip, from side view images at different speeds (cf. inset of Fig. 3). 
Strictly speaking, (2) is derived for straight contact lines. We therefore perform our measurements at a distance $\ll R$, where the effect of contact line curvature should be negligible. Given the resolution of the side view images, we take $x=50 \mu \mathrm{m}$ in order to have sufficient accuracy on the contact angle.

Figure 3 shows $\theta^{3}$ versus Ca. We clearly distinguish the linear regime of (2), as well as a departure from this behavior at higher drop speeds. From a linear fit we recover the (receding) equilibrium angle $\theta_{e}=45 \pm 1^{\circ}$ as well as $\ell_{\theta}=8 \pm 5 \mathrm{~nm}$. This length scale is consistent with the order of magnitude found from the $R$ measurements. The deviation from the Cox-Voinov behavior occurs when the radius of curvature approaches the measurement scale of $50 \mu \mathrm{m}$, around $\mathrm{Ca} \approx 6 \cdot 10^{-3}$. This once more suggests an interaction between $R$ and $\ell_{\theta}$ in the corner regime.

Lubrication model. - We interpret these findings within a lubrication model that incorporates the strongly curved tip. For small contact angles the shape of the liquid-gas interface, $h(x, y)$, obeys a partial differential equation that expresses a balance between capillary and viscous forces [32]. The multi-scale nature of the problem makes it difficult to solve the equation by direct simulation. Instead, we propose an approximate analysis that has the additional advantage of yielding expressions in closed form. First, we assume that the flow is oriented purely in the $x$ direction (Fig. 4 , inset), so that the lubrication equation simplifies

$$
\partial_{x} \kappa=\frac{3 \mathrm{Ca}}{h^{2}} .
$$

$\kappa$ is the interface curvature providing the Laplace pressure $p=-\gamma \kappa$. For sharp corners with vanishing tip size, this 'planar flow approximation' was found very accurate [21]. In the present case this corresponds to $x \gg R$. For $x \ll R$ the flow becomes truly one-dimensional since $\partial / \partial_{y} \ll \partial / \partial_{x}$ and (3) is even exact. In this region $\kappa=\partial_{x x} h$, and (3) can be integrated to the Cox-Voinov law $[23,24,33]$. For corners such as in Fig. 1 we thus expect to recover (2) at small distance from the tip.

Away from the tip, $x \sim R$, the interface exhibits a truly two-dimensional structure, $\kappa=\partial_{x x} h+\partial_{y y} h$, making the analysis much more involved. This effect has been considered in the limit of weak contact line curvature [34], but this is not sufficient for the present purpose. To make progress we estimate $\kappa$ by (i) approximating the contact line shape by a hyperbola, $y_{\mathrm{cl}}^{2}=2 R x+\phi^{2} x^{2}$, and (ii) approximating the cross-section of the corner by a parabola. The quality of the former approximation can be inferred from the dashed line of Fig. 1d, while the latter has been justified in detail in [21]. With this, the interface is parameterized as (cf. inset Fig. 4)

$$
h(x, y)=h_{0}(x)\left(1-\frac{y^{2}}{y_{\mathrm{cl}}^{2}}\right)
$$

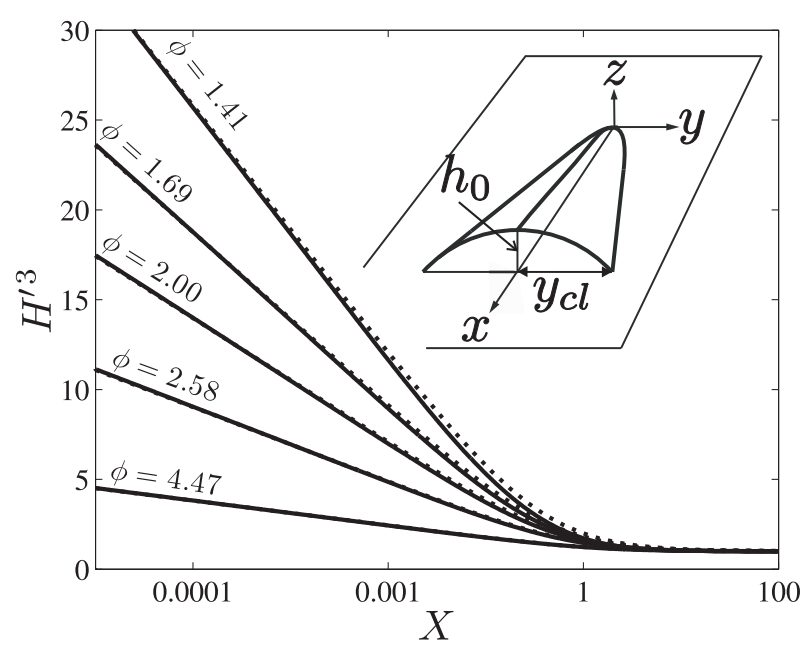

FIG. 4: Rescaled slope $H^{\prime 3}$ obtained from numerical solution of (6) with boundary conditions $H^{\prime}(\infty)=1, H(0)=0$ (solid lines). The Cox-Voinov logarithmic variation saturates at a distance $X \sim 1$, corresponding to the tip radius. Dotted lines represent (7) using $\beta$ as fit parameter. Inset: the drop shape is modeled by a hyperbolic contact line shape $y_{\mathrm{cl}}(x)$ and parabolic cross section. The centerline profile $h_{0}(x)$ can then be computed from (6).

containing $R$ and $\phi$, the opening angle of the hyperbola, as parameters. In addition, we still need to solve the centerline profile $h_{0}(x)$. To close the problem we evaluate the curvature at the centerline $y=0$,

$$
\kappa=h_{0}^{\prime \prime}-\frac{2 h_{0}}{2 R x+\phi^{2} x^{2}},
$$

which together with (3) provides an ordinary differential equation for $h_{0}(x)$. This equation was previously studied with vanishing tip radius $R=0[21,22]$, here representing the limit $x \gg R$. This regime admits solutions with a well-defined corner angle $h_{0}^{\prime}(\infty)=\Omega=\left(3 \mathrm{Ca} \phi^{2} / 2\right)^{1 / 3}$.

It is convenient to introduce dimensionless variables $X=x \phi^{2} / R$ and $H=h_{0} \phi^{2} / R \Omega$, so that from $(3,5)$ we obtain the equation on the centerline

$$
\frac{\phi^{2}}{2} H^{\prime \prime \prime}-\left(\frac{H}{X(X+2)}\right)^{\prime}=\frac{1}{H^{2}}
$$

The only remaining parameter is the opening angle $\phi[35]$. We have the asymptotic boundary conditions $H^{\prime}(\infty) \rightarrow 1$ towards the corner and $H(0) \rightarrow 0$ towards the contact line. Figure 4 displays the solutions obtained from numerical integration, for various $\phi$. At small $X$ one recovers the Cox-Voinov logarithmic variation of the slope $H^{\prime}$, showing up as a straight line on this plot. However, this trend saturates at large $X$, when the two-dimensional nature of the curvature becomes apparent. All solutions are very accurately represented by the form 


$$
H^{\prime 3}=1+\frac{6}{\phi^{2}} \ln \left(1+\frac{\beta}{X}\right),
$$

as can be seen from Fig. 4 (dotted lines). We used $\beta$ as a fit parameter that turns out to depend weakly on $\phi$. A perturbation expansion shows that (7) is in fact the exact solution for $\phi \gg 1$, with $\beta=2$.

To solve for the tip radius, the final step is to match (2) to the small scale asymptote of (7), which in original variables reads $h^{\prime 3} \simeq \Omega^{3}+9 \mathrm{Ca} \ln \frac{\beta R}{x \phi^{2}}$. Equating the two expressions one finds

$$
\Omega^{3}=\theta_{e}^{3}-9 \mathrm{Ca} \ln \frac{\beta R}{\phi^{2} \ell_{\theta}},
$$

which is the sought for relation between $R$, Ca and the contact angles. Since in practice $\Omega \ll \theta_{e}$, it can be recast as (1) with $\ell=\ell_{\theta} \phi^{2} / \beta$. Strictly speaking this length contains a dependence on drop speed through the opening angle $\phi$ that also induces a variation of $\beta$. This variation, however, is subdominant with respect to the exponential dependence in (1).

Let us emphasize that the structure (7) is robust with respect to the choice of parameterization of the interface shape and the 'planar flow approximation', since these only affect the cross-over to the corner regime. On the other hand, the numerical value of $\beta$ is determined from the second term in (5) and will certainly be model dependent. One should bear in mind that these details fall within the experimental uncertainty on $\ell$ and $\ell_{\theta}$.

Outlook. - We have identified a new kind of singularity in free surface flows for which the regularization involves a microscopic (inner) scale instead of a macroscopic (outer) scale. In fact, the corner is obtained by sharply bending the line singularity associated to the viscous divergence near the contact line. This is very specific for wetting flows and differs qualitatively from other free surface singularities. Our findings also emphasize that the dynamic contact angle is strongly affected by the corner. This gives a departure of the Cox-Voinov behavior when the tip size becomes comparable to the scale of measurement. It would be interesting to compare these results to advancing contact lines, where bubble entrainment occurs through sharp corners as well [8-10].

In the experiment, the minimum tip size that can be achieved is limited by the onset of the pearling instability. This instability can possibly be incorporated in the model by matching the cross sections to the inclined contact lines, along the lines of Ref. [22]. In that study, however, the tip radius was neglected and incorrectly predicted a vanishing size of emitted drops at threshold. In practice these drops are or the order of $100 \mu \mathrm{m}$, which we speculate to be related to the finite radius of the tip.

IP acknowledges financial support by NWO.
[2] R. F. Day, E. J. Hinch, and J. R. Lister, Phys. Rev. Lett. 80, 704 (1998).

[3] J.-T. Jeong and H. K. Moffatt, J. Fluid Mech. 241, 1 (1992).

[4] J. Eggers, Phys. Rev. Lett. 86, 4290 (2001).

[5] I. Cohen and S. R. Nagel, Phys. Rev. Lett. 88, 074501 (2002).

[6] W. W. Zhang, Phys. Rev. Lett. 93, 184502 (2004).

[7] S. Courrech du Pont and J. Eggers, Phys. Rev. Lett. 96, 034501 (2006).

[8] R. Burley and B. S. Kennedy, Chem. Eng. Sci. 31, 901 (1976).

[9] T. D. Blake and K. J. Ruschak, Nature 282, 489 (1979).

[10] H. Benkreira and M. I. Khan, Chem. Eng. Sci. 63, 448 (2008).

[11] E. Lorenceau, D. Quéré, and J. Eggers, Phys. Rev. Lett 93, $254501(2004)$

[12] E. Lorenceau, F. Restagno, and D. Quéré, Phys. Rev. Lett. 90, 184501 (2003).

[13] A. M. Gañán-Calvo, Phys. Rev. Lett. 79, 217 (1997).

[14] J. Eggers and E. Villermaux, Rep. Prog. Phys. 71, 036601 (2008)

[15] S. F. Kistler and P. Schweizer, Liquid film coating - Scientific principles and their technological implications, vol. 169 (Kluwer Academic, Dordrecht, 1997).

[16] T. Podgorski, J. M. Flesselles, and L. Limat, Phys. Rev. Lett. 87, 036102(1) (2001).

[17] N. Le Grand, A. Daerr, and L. Limat, J. Fluid Mech. 541, 293 (2005).

[18] C. Duez, C. Ybert, C. Clanet, and L. Bocquet, Nat. Phys. 3, 180 (2007).

[19] C. Huh and L. E. Scriven, J. Coll. Int. Sci. 35, 85 (1971).

[20] E. B. Dussan V. and S. H. Davis, J. Fluid Mech. 65, 71 (1974).

[21] L. Limat and H. A. Stone, Europhys. Lett. 65, 365 (2003).

[22] J. H. Snoeijer, N. Le Grand-Piteira, L. Limat, H. A. Stone, and J. Eggers, Phys. Fluids 19, 042104 (2007).

[23] O. V. Voinov, Fluid Dynamics 11, 714 (1976)

[24] R. G. Cox, J. Fluid Mech. 168, 169 (1986).

[25] P. G. de Gennes, Coll. Pol. Sci. 264, 463 (1986).

[26] Y. Pomeau, C.R. Acad. Sci. Paris 328, 411 (2000).

[27] J. Eggers, Phys. Rev. Lett. 93, 094502 (2004).

[28] U. Thiele, K. John, and M. Bär, Phys. Rev. Lett. 93, $027802(2004)$.

[29] L. W. Schwartz, D. Roux, and J. J. Cooper-White, Physica D 209, 236 (2005).

[30] E. Rolley and C. Guthman, Phys. Rev. Lett. 98, 166105 (2007).

[31] J. De Coninck and T. D. Blake, Annu. Rev. Mater. Res. 38, 1 (2008).

[32] A. Oron, S. H. Davis, and S. G. Bankoff, Rev. Mod. Phys. 69, 931 (1997)

[33] B. R. Duffy and S. K. Wilson, Appl. Math. Lett. 63, 63 (1997).

[34] J. H. Snoeijer, E. Rio, N. Le Grand, and L. Limat, Phys. Fluids 17, 072101 (2005).

[35] Experimentally, the angle $\phi$ decreases with drop speed, in order to reduce the normal velocity of the inclined contact lines $[16,17]$; its value remains of order unity. 